\title{
Weak Supervision for Fake News Detection via Reinforcement Learning
}

\author{
Yaqing Wang, ${ }^{1}$ Weifeng Yang, ${ }^{2}$ Fenglong Ma, ${ }^{3}$ Jin Xu, ${ }^{2 *}$ Bin Zhong, ${ }^{2}$ Qiang Deng, ${ }^{2}$ Jing Gao ${ }^{1 *}$ \\ ${ }^{1}$ State University of New York at Buffalo, New York, USA \\ ${ }^{2}$ Data Quality Team, WeChat, Tencent Inc., China \\ ${ }^{3}$ Pennsylvania State University, Pennsylvania, USA \\ ${ }^{1}$ yaqingwa, jing $\} @$ buffalo.edu, ${ }^{2}$ \{curryyang, jinxxu, harryzhong, calvindeng $\} @$ tencent.com, ${ }^{3}$ fenglong@ @su.edu
}

\begin{abstract}
Today social media has become the primary source for news. Via social media platforms, fake news travel at unprecedented speeds, reach global audiences and put users and communities at great risk. Therefore, it is extremely important to detect fake news as early as possible. Recently, deep learning based approaches have shown improved performance in fake news detection. However, the training of such models requires a large amount of labeled data, but manual annotation is time-consuming and expensive. Moreover, due to the dynamic nature of news, annotated samples may become outdated quickly and cannot represent the news articles on newly emerged events. Therefore, how to obtain fresh and high-quality labeled samples is the major challenge in employing deep learning models for fake news detection. In order to tackle this challenge, we propose a reinforced weaklysupervised fake news detection framework, i.e., WeFEND, which can leverage users' reports as weak supervision to enlarge the amount of training data for fake news detection. The proposed framework consists of three main components: the annotator, the reinforced selector and the fake news detector. The annotator can automatically assign weak labels for unlabeled news based on users' reports. The reinforced selector using reinforcement learning techniques chooses highquality samples from the weakly labeled data and filters out those low-quality ones that may degrade the detector's prediction performance. The fake news detector aims to identify fake news based on the news content. We tested the proposed framework on a large collection of news articles published via WeChat official accounts and associated user reports. Extensive experiments on this dataset show that the proposed WeFEND model achieves the best performance compared with the state-of-the-art methods.
\end{abstract}

\section{Introduction}

The recent proliferation of social media has significantly changed the way in which people acquire information. According to the 2018 Pew Research Center survey, about twothirds of American adults (68\%) get news on social media at least occasionally. Fake news, which refer to intentionally and verifiably false news stories, can spread virally on

\footnotetext{
${ }^{*}$ Corresponding authors. Copyright (C) 2020, Association for the Advancement of Artificial Intelligence (www.aaai.org). All rights reserved.
}

social media platforms as people rarely verify the source of the news when sharing a news article that sounds true. The spread of fake news may bring many negative impacts, including social panic and financial loss. Recent years have witnessed a number of high-impact fake news spread regarding terrorist plots and attacks, presidential election, and various natural disasters. In many of these cases, even when correct information later disseminates, the rapid spread of fake news can have devastating consequences. Therefore, there is an urgent need for the development of automatic fake news detection algorithms which can detect fake news as early as possible and help stop the viral spread of such news.

Recently, many approaches are proposed to identify fake news, which can be roughly divided into two categories, i.e., traditional learning (Conroy, Rubin, and Chen 2015; Tacchini et al. 2017) and deep learning based models (Ruchansky, Seo, and Liu 2017; Ma et al. 2016; Wang et al. 2018; Popat et al. 2018). Traditional learning methods typically extract features from news articles and train classifiers based on the extracted features. Compared with traditional learning methods, deep learning models have achieved an improvement in the performance of fake news detection due to their powerful abilities of learning informative representations automatically. However, training deep learning models usually requires a large amount of hand-labeled data, i.e., news articles that are labeled as real or fake. The creation of such data is expensive and time-consuming. Also, accurate labels can only be obtained when the annotators have sufficient knowledge about the events. Furthermore, the dynamic nature of news articles leads to decaying quality of existing labeled samples. Some of these samples may become outdated quickly and cannot represent the news articles on newly emerged events. To maintain the quality of labeled samples, annotators have to continuously label newly emerging news articles, which is infeasible. To fully unleash the power of deep learning models in fake news detection, it is essential to tackle the challenge of labeling fake news.

A possible solution is to leverage the feedback provided by users who read the news. Nearly every social medial platform provides a way for users to report their comments about the news, and some of these comments are highly relevant to fake news detection. For example, for a news article pub- 
lished on a WeChat official account ${ }^{1}$, a user who reads the article can report whether this news is fake or not with a brief explanation. Such reports from users can be regarded as "weak" annotation for the task of fake news detection. The large collection of user reports can help alleviate the label shortage problem in fake news detection. However, different from expert-labeled samples, these weak annotated samples are unavoidably noisy. Users may report real news as fake ones, and the reasons they provide may not be meaningful. Therefore, how to transform weak annotation to labeled samples in the training set and select high-quality samples is the major issue we need to solve.

In light of the aforementioned challenges, we propose a reinforced WEakly-supervised FakE News Detection framework (WeFEND), which can leverage the crowd users' feedback as weak supervision for fake news detection. The proposed framework WeFEND consists of three main components: the annotator, the fake news detector and the reinforced selector. In particular, given a small set of labeled fake news samples together with users' feedback towards these news articles, we can train an annotator based on the feedback, which can then be used to automatically assign weak labels for those unlabeled news articles simply based on the user feedback they received. The reinforced selector which employs reinforcement learning techniques then selects high-quality samples from weakly labeled samples as the input to the fake news detector. The fake news detector finally assigns a label for each input article based on its content. The three components integrate nicely and their interactions mutually enhance their performance. We conduct extensive experiments on a large collection of news articles published by WeChat official accounts and corresponding feedback reported by users on these articles. Experimental results show that the proposed framework WeFEND outperforms the state-of-the-art approaches on fake news detection. Moreover, we will publicly release this dataset ${ }^{2}$ to the community to encourage further research on fake news detection with user reports.

The main contributions of this paper can be summarized as follows:

- We recognize the label shortage issue and propose to leverage user reports as weak supervision for fake news detection on news content. Towards this end, we propose an effective weakly-supervised fake news detection framework.

- The proposed WeFEND framework can automatically annotate news articles, which help enlarge the size of the training set to ensure the success of deep learning models in fake news detection.

- Adopting reinforcement learning techniques, the proposed framework WeFEND has the ability of selecting high-quality samples, which further leads to the improvement of the fake news detection performance.

\footnotetext{
${ }^{1}$ WeChat is a Chinese multi-purpose messaging, social media and mobile payment app developed by Tencent. Wechat official accounts push news articles and information for subscribed followers.

${ }^{2}$ https://github.com/yaqingwang/WeFEND-AAAI20
}

- We empirically show that the proposed framework WeFEND can effectively identify fake news and significantly outperform the state-of-the-art fake news detection models on a large-scale dataset collected from WeChat official accounts.

\section{Related Work}

In this section, we briefly review the work related to the proposed model. We mainly focus on the following two topics: fake news detection and reinforcement learning.

There are many tasks related to fake news detection, such as rumor detection (Jin et al. 2014) and spam detection (Shen et al. 2017). Following the previous works (Ruchansky, Seo, and Liu 2017; Shu et al. 2017), we specify the definition of fake news as news which is intentionally fabricated and can be verified as false. Many fake news detection algorithms try to distinguish news according to their features, which can be extracted from social context and news content.

Social context features represent the user engagements of news on social media (Shu et al. 2017) such as the number of followers, hash-tag(\#) retweets and the network structure(Wu, Yang, and Zhu 2015). However, social context features can only be extracted after an accumulated period of time, and thus cannot be used in a timely detection of newly emerged fake news.

News content features are statistical or semantic features extracted from the textual content of news, which has been explored in many literatures of fake news detection (Gupta et al. 2014; Kwon et al. 2013; Castillo, Mendoza, and Poblete 2011). It is difficult to design hand-crafted textual features for traditional machine learning based fake news detection models. To overcome this limitation, Ma et al. (Ma et al. 2016) and Wang et al. (Wang et al. 2018) proposed deep learning models to identify fake news based on news text and multi-modal data respectively. These models have shown the improvement in detection performance, but the power of deep learning models are not yet fully unleashed due to the lack of fresh high-quality samples for training.

The manual labeling of news articles is expensive to obtain, so user feedback, which is valuable signal for fake news detection, should be incorporated into the detection process. However, feedback by users may not be reliable and user reliability is unknown. The power-law distribution of user participation rates makes it difficult to correctly estimate users' reliability (Moore and Clayton 2008; Chia and Knapskog 2011). In recent work (Tschiatschek et al. 2018; Kim et al. 2018), such crowd signals were used in fake news detection. From those news articles that are flagged by users as potential fake news, these methods select a small subset and send them to experts for confirmation. Therefore, they still require manual labeling, and valuable feedback comments are not taken into consideration.

In fact, when users flag the suspicious articles, the social media platforms usually require users to provide brief explanations on why the news is fake. Such comments provide important information for the fake news detection task. Different from the existing works, we propose to incorporate these informative explanations into the detection model. More specifically, the proposed framework WeFEND can 
leverage report messages as weak supervision to guide fake news detection based on news content.

To further improve the detection performance, we incorporate reinforcement learning techniques (Sutton and Barto 1998) into the proposed framework to select high-quality samples. Reinforce learning was adopted in (Feng et al. 2018; Wu, Li, and Wang 2018) to learn an instance selector based on the prediction probabilities, but our selection approach is quite different. As the distribution of news articles is changing over time, prediction probabilities are not suitable as evaluation criteria for data selection in our problem setting. Therefore, to select high quality data for fake news detection, we propose a novel performance-driven data selection method based on reinforcement learning mechanism.

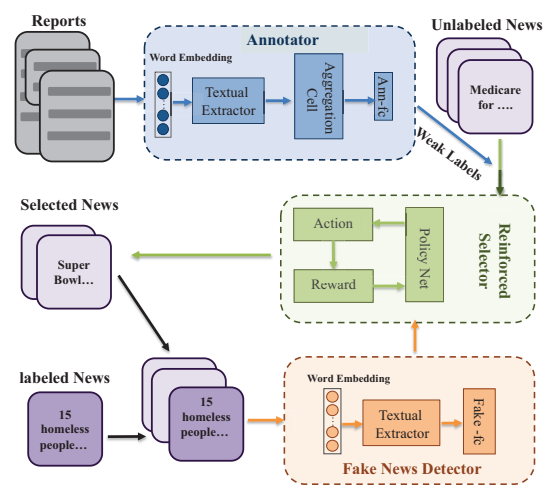

Figure 1: The architecture of proposed framework WeFEND which consists of annotator, reinforced selector and fake news detector.

\section{Methodology}

In this section, we first briefly introduce the overview of the proposed fake news detection framework WeFEND, and then demonstrate each component in detail.

\section{Overview}

The problem setting is as follows. Each sample consists of both news articles and user feedback comments. Both are texts, and are transformed into vector representations by word embedding. User feedback comments are referred to as reports, which are detailed reasons and evidence provided by users about the credibility of the corresponding news articles. A small set of samples are labeled by experts as fake or real, and our objective is to predict the labels of the unlabeled samples.

Figure 1 shows the overview of the proposed framework WeFEND. There are three key components: annotator, data selector and fake news detector. Annotator can be seen as a pretrained model on the reports with their labels. Based on the pretrained model, we can assign weak labels for the unlabeled samples according to the annotator on the reports. However, it is hard to guarantee the quality of weak labels. To automatically choose high-quality samples, we design a data selector by exploiting reinforcement learning techniques on the samples labeled by the annotator. Finally, the selected samples and the original labeled samples are used to train fake news detector. In both annotator and fake news detector, a textual feature extractor is used to extract features from input text. The details of these components are introduced in the following subsections.

\section{Textual Feature Extractor}

From Figure 1, we can observe that textual feature extractor is a basic module of annotator and fake news detection, as not all words are relevant to the task of fake news detector. In this paper, we choose convolutions neural network (Kim 2014), which is proven effective in the fake news detection (Wang et al. 2018), as textual feature extractor. The input of the textual feature extractor is news content or a report message, and both can be represented as a sequential list of words. For the $t$-th word in the sentence, we represent it by the corresponding $d$ dimensional word embedding vector, denoted as $\mathbf{x}_{t} \in \mathbb{R}^{d}$, which is the input to the convolutions neural network. Details of CNN module (Kim 2014) are in the Supplemental Material.

The learned representation from textual feature extractor are the input features to annotator and fake news detector. Next, we will introduce how to train an annotator and use it to assign weak labels to the unlabeled samples.

\section{Automatic Annotation based on Reports}

One benefit of the proposed framework is that it can automatically assign weak labels to the unlabeled news samples, which helps enlarge the size of the training set with little costs. To train such a model, we propose to use report messages provided by users as weak supervision.

Aggregation Cell. One news article may have reports from multiple users, so we propose to aggregate features obtained from different reports for one sample. Since the report messages from multiple users for one piece of news are permutation invariant, we design an aggregation cell consisting of a commutative aggregation function and a fullyconnected layer. The commutative aggregation function, like sum, mean and max-pooling, can combine the permutation invariant input set. We take the $i$-th sample as an example, and the $j$-th report message is represented as $r_{j}^{(i)}$. The corresponding report message set is denoted as $R^{(i)}=$ $\left\{r_{1}^{(i)}, r_{2}^{(i)}, \ldots, r_{\left|R^{(i)}\right|}^{(i)}\right\}$, where $\left|R^{(i)}\right|$ is the number of report messages of the $i$-th sample. The report message $r_{j}^{(i)} \in R^{(i)}$ is first fed into the textual feature extractor to obtain an informative textual feature representation, denoted as $\mathbf{h}_{j}^{(i)}$. Then we use the aggregation cell to combine the report message set $R^{(i)}$ to learn the hidden feature representation $\mathbf{h}^{(i)}$. In order to stabilize the training procedure, we use average operation as the commutative aggregation function. The procedure of aggregation cell is represented as:

$$
\mathbf{h}^{(i)}=\operatorname{ReLU}\left(\mathbf{w}_{r} \cdot \sum_{j=1}^{\left|R^{(i)}\right|} \frac{\mathbf{h}_{j}^{(i)}}{\left|R^{(i)}\right|}\right),
$$

where $\mathbf{w}_{r}$ is the weight of the fully-connected layer. 
We feed $\mathbf{h}^{(i)}$ into the fully connected layer, denoted as Ann-fc, to output the corresponding probability of the $i$-th sample being a fake one, which is denoted as $D_{r}\left(R^{(i)}, \theta_{r}\right)$, where $\theta_{r}$ represents all the parameters of the annotator and corresponding textual feature extractor. The entire report message dataset is represented as $R=$ $\left\{R^{(1)}, R^{(2)}, \ldots, R^{(|R|)}\right\}$, and the corresponding ground truth labels of news are denoted as $Y=\left\{y^{(1)}, y^{(2)}, \ldots, y^{(|R|)}\right\}$, where $|R|$ is the number of report sets. Based on $R$ and $Y$, the loss function for the proposed annotator is defined by cross entropy as follows:

$$
\begin{aligned}
L_{r}\left(R, Y ; \theta_{r}\right)= & -\frac{1}{|R|} \sum_{i=1}^{|R|}\left[y^{(i)} \log D_{r}\left(R^{(i)} ; \theta_{r}\right)\right. \\
& \left.+\left(1-y^{(i)}\right) \log \left(1-D_{r}\left(R^{(i)} ; \theta_{r}\right)\right)\right] .
\end{aligned}
$$

Given the unlabeled news set $X^{u}$ with corresponding report messages, we use the trained annotator to predict their labels, which are denoted as $\hat{Y}^{u}$. By the annotator, we can obtain a large weakly labeled dataset $\left\{X^{u}, \hat{Y}^{u}\right\}$. However, the labels in this automatically-annotated dataset are unavoidably noisy and directly adding these samples to the training set may degrade the detection performance. Thus, the challenge here is how to select high-quality samples from this set to guarantee the detection performance. To address this challenge, we propose to employ reinforcement learning techniques in the design of a data selector. The details of the proposed data selector are introduced in the following subsection.

\section{Data Selection via Reinforcement Learning}

The objective of the data selector is to automatically select high-quality samples from those with weak labels obtained from the annotator. The criteria of the selection is based on whether adding the chosen sample can improve the fake news detection performance. According to this criteria, we design a performance-driven data selection method (called reinforced data selector) using reinforcement learning mechanism. Next, we first introduce the input data of the designed data selector, and then present the details of this data selector.

Let $\tilde{X}$ denote all the input data of the proposed reinforced data selector. However, instead of directly putting the entire dataset $\tilde{X}$ into the selector, we divide the whole dataset into $K$ small bags of data samples, i.e., $\tilde{X}=\left\{\tilde{X}^{(k)}\right\}_{k=1}^{K}$. A bag of data samples is the input of the selector each time. For the $k$-th bag of data $\tilde{X}^{(k)}$, it contains $B$ samples, i.e., $\tilde{X}^{(k)}=\left\{x_{1}^{(k)}, x_{2}^{(k)}, \ldots, x_{B}^{(k)}\right\}$. The benefit of using multiple small bags of samples is that this approach can provide more feedback to the selector and this makes the training procedure of reinforcement learning more efficient.

Problem Formulation. In the data selection procedure, the samples in one bag are sequentially fed into the designed reinforced data selector. For every sample, the action of reinforced data selector is to retain or remove. The decision of the current sample $x_{i}^{(k)}$ is based on its state vector and all the previous decisions of samples $\left\{x_{1}^{(k)}, x_{2}^{(k)}, \ldots, x_{i-1}^{(k)}\right\}$. Thus, the data selection problem can be naturally cast as a Markov Decision Process (MDP) that is the prerequisite of reinforcement learning. Since the goal of data selection is to improve the performance of fake news detection, we directly use the performance changes of fake news detection as the reward for reinforced selector. The performance is evaluated by accuracy. For this sequential decision procedure, the reward is delayed because it can only be obtained after all the decisions are made. To solve the delayed reward problem, we employ the policy-based reinforcement learning mechanism. Since the reinforced selector needs to use the performance changes of fake news detection as reward, we introduce the fake news detector first.

Fake News Detector. Fake news detection model is a neural network, which consists of a textual feature extractor and a fully-connected layer, namely Fake-fc, with corresponding activation functions. The input to fake news detector is news content, and the output is the probability of the given news being fake. The detector is denoted as $D_{n}\left(\cdot ; \theta_{n}\right)$, where $\theta_{n}$ represents all the parameters.

After introducing fake news detector, we will introduce the concepts of state, action, and reward used in the proposed reinforced selector in detail as follows.

State. The state vector of the sample $x_{i}^{(k)}$ is denoted as $s_{i}^{(k)}$. Since every action is made based on the current sample and the chosen sample, the state vector mainly consists of two components: the representation of the current sample and the average representation of the chosen samples. The representation of the current sample is related to data quality and diversity. We use the output probability from the proposed annotator and the output probability of fake news detector to measure the quality of the data. To represent the data diversity, we first calculate cosine similarity between the current sample and all the chosen samples. Here each sample is represented by a vector obtained from textural feature extractor. We then select the max value of cosine similarity as the diversity. To balance the distribution of classes, the weak label of the current sample is also used as a part of the representation. Therefore, the current state vector contains four elements: 1) the output probability from the annotator, 2) the output probability from fake news detector, 3 ) the maximum of cosine similarity between the current sample and the chosen samples, and 4) the weak label of the current sample. The representations of all the chosen samples are defined as the average of all the chosen samples' state vectors. The concatenation of the current state vector and the average of previous state vectors is considered as the final state vector $s_{i}^{(k)}$.

Action. The action value of the reinforced selector for every sample is 1 or 0.1 represents the action to retain the sample, and 0 denotes the action to remove it. To determine the action, we train a policy network, denoted as $P\left(\cdot ; \theta_{s}\right)$, where $\theta_{s}$ represents the parameters. The policy network includes two fully connected layers with corresponding activation functions. Take the sample $x_{i}^{(k)}$ as an example. The policy network outputs a probability of retaining, denoted as 
$p_{i}^{(k)}$, based on the sample's state vector $s_{i}^{(k)}$ :

$$
P\left(s_{i}^{(k)} ; \theta_{s}\right)=\delta\left(\mathbf{w}_{s 2} \cdot \operatorname{ReLU}\left(\mathbf{w}_{s 1} \cdot s_{i}^{(k)}\right)\right),
$$

where $\mathbf{w}_{s 1}$ and $\mathbf{w}_{s 2}$ are the weights of two fully-connected layers and $\delta$ represents the Sigmoid activation function. Then the action $a_{i}^{(k)}$ is sampled according to the output probability. The policy $\pi_{\theta_{s}}\left(s_{i}^{(k)}, a_{i}^{(k)}\right)$ can be represented as

$$
\pi_{\theta_{s}}\left(s_{i}^{(k)}, a_{i}^{(k)}\right)=\left\{\begin{array}{ll}
p_{i}^{(k)} & \text { if } a_{i}^{(k)}=1 \\
1-p_{i}^{(k)} & \text { if } a_{i}^{(k)}=0
\end{array} .\right.
$$

Reward. Since the goal of the action is to retain the samples that can bring improvement to fake news detection, we use the performance changes of detection model $D_{n}\left(\cdot ; \theta_{n}\right)$ as the reward function. Given the $k$-th bag of data $\left\{x_{1}^{(k)}, x_{2}^{(k)}, \ldots, x_{B}^{(k)}\right\}$, the actions of retaining or removing are made based on the probability output from the policy network. To evaluate the performance changes, we need to set a baseline accuracy acc. Towards this end, we first extract a validation dataset from the whole labeled dataset. Note that all the trained model will test on this extracted validation dataset. We then calculate the baseline accuracy acc with the detection model $D_{n}\left(\cdot ; \theta_{n}\right)$. Since the designed data selector can choose some high-quality samples from $\left\{x_{1}^{(k)}, x_{2}^{(k)}, \ldots, x_{B}^{(k)}\right\}$, the fake news detection model will be retrained using the retained data samples. A new accuracy $a c c_{k}$ can be obtained with the retrained model on the validation dataset. Finally, the reward $R_{k}$ for $k$-th bag data $\left\{x_{i}^{(k)}\right\}_{i=1}^{B}$ is represented by the difference of $a c c_{k}$ and $a c c$ as follows:

$$
R_{k}=a c c_{k}-a c c .
$$

For the $k$-th bag of data $\left\{x_{i}^{(k)}\right\}_{i=1}^{B}$, we aim to maximize the expected total reward. Since the scale of $R_{k}$ is small, we use the summation of reward to define the objective function in order to make the training procedure more efficient. The objective function is defined as

$$
J\left(\theta_{s}\right)=\sum_{i=1}^{B} \pi_{\theta_{s}}\left(s_{i}^{(k)}, a_{i}^{(k)}\right) R_{k} .
$$

The derivative of the objective function above is

$$
\begin{aligned}
\nabla_{\theta} J\left(\theta_{s}\right) & =\sum_{i=1}^{B} R_{k} \nabla_{\theta_{s}} \pi_{\theta_{s}}\left(s_{i}^{(k)}, a_{i}^{(k)}\right) \\
& =\mathbb{E}_{\pi_{\theta_{s}}}\left[\sum_{i=1}^{B} R_{k} \nabla_{\theta_{s}} \log \pi_{\theta_{s}}\left(s_{i}^{(k)}, a_{i}^{(k)}\right)\right]
\end{aligned}
$$

According to the policy-based reinforcement learning algorithm (Sutton et al. 2000; Sutton and Barto 1998), we update the parameters $\theta$ of the policy network by stochastic gradient ascent as follows:

$$
\theta_{s} \leftarrow \theta_{s}+\alpha \sum_{i=1}^{B} R_{k} \nabla_{\theta_{s}} \log \pi_{\theta_{s}}\left(s_{i}^{(k)}, a_{i}^{(k)}\right),
$$

where $\alpha$ is the learning rate. To improve the exploration and stabilize training, we train a target policy network $P\left(\cdot, \theta_{s}{ }^{\prime}\right)$ that updates much slower than the policy network $P\left(\cdot, \theta_{s}\right)$ :

$$
\theta_{s}^{\prime}=(1-\tau) \theta_{s}^{\prime}+\tau \theta_{s}
$$

In the training stage, half of the bags are fed into the policy network $P\left(\cdot ; \theta_{s}\right)$ and the another half of bags are fed into the target policy network $P\left(\cdot ; \theta_{s}^{\prime}\right)$.

\section{Reinforced Weakly-supervised Fake News Detection Framework}

In this subsection, we introduce how to integrate the three key components: annotator, fake news detector and reinforced selector. First, we pretrain the annotator using the labeled report data $\{R, Y\}$ and assign weak labels $\hat{Y}^{u}$ to the unlabeled news set $X^{u}$. The proposed reinforced selector will select high-quality samples from the weakly labeled dataset $\left\{X^{u}, \hat{Y}^{u}\right\}$. Here we set the selected bags as $K$. Then both the selected data set $\left\{X_{s}, Y_{s}\right\}=\left\{X_{s}^{(k)}, Y_{s}^{(k)}\right\}_{k=1}^{K}$ and the original labeled data are fed into the fake news detector for training. Thus, the final loss of fake news detection consists of two sub losses:

$$
\begin{aligned}
L_{n}\left(X, Y, X_{s}, Y_{s} ; \theta_{n}\right)= & \lambda_{l} \cdot L_{n}^{l}\left(X, Y ; \theta_{n}\right) \\
& +\lambda_{s} \cdot L_{n}^{s}\left(X_{s}, Y_{s} ; \theta_{n}\right),
\end{aligned}
$$

where $L_{n}^{l}\left(X, Y ; \theta_{n}\right)$ and $L_{n}^{s}\left(X_{s}, Y_{s} ; \theta_{n}\right)$ are the losses on a small amount of manually labeled data and automaticallyannotated data set respectively. Here the $\lambda_{l}$ and $\lambda_{u}$ control the balance between $L_{n}^{l}\left(\theta_{n}\right)$ and $L_{n}^{s}\left(\theta_{n}\right)$, and we simply set the values of $\lambda_{l}$ and $\lambda_{u}$ as 1 . The two losses are defined by cross entropy respectively as follows:

$$
\begin{aligned}
L_{n}^{l}\left(X, Y ; \theta_{n}\right)= & -\mathbb{E}_{(x, y) \sim(X, Y)}\left[y \log \left(D_{n}\left(x ; \theta_{n}\right)\right)\right. \\
& \left.+(1-y) \log \left(1-D_{n}\left(x ; \theta_{n}\right)\right)\right], \\
L_{n}^{s}\left(X_{s}, Y_{s} ; \theta_{n}\right)= & -\mathbb{E}_{\left(x_{s}, y_{s}\right) \sim\left(X_{s}, Y_{s}\right)}\left[y_{s} \log \left(D_{n}\left(x_{s}, \theta_{n}\right)\right)\right. \\
& \left.+\left(1-y_{s}\right) \log \left(1-D_{n}\left(x_{s} ; \theta_{n}\right)\right)\right] .
\end{aligned}
$$

\section{Experiments}

In this section, we introduce the dataset used in the experiments, present the compared fake news detection models, validate the effectiveness and explore some insights of the proposed framework.

\section{Dataset}

To fairly evaluate the performance of the proposed framework, we collect a dataset from WeChat's Official Accounts and conduct comprehensive experiments to analyze the performance. This dataset includes user reports and will be publicly released in future to encourage research on fake news detection. In this dataset, the news are collected from WeChat's Official Accounts, dated from March 2018 to October, 2018. To facilitate the detection fake news, the WeChat's Official Account encourages users to report suspicious articles, and write feedback to explain why they think 
Table 1: The Statistics of the WeChat Datasets.

\begin{tabular}{c|c|c|c|c}
\hline & & \# News & \# Report & \# Avg. Reports/News \\
\hline Unlabeled & - & 22981 & 31170 & 1.36 \\
\hline \multirow{2}{*}{ Labeled Training } & Fake & 1220 & 2010 & 1.65 \\
\cline { 2 - 5 } & Real & 1220 & 1740 & 1.43 \\
\hline \multirow{2}{*}{ Labeled Testing } & Fake & 870 & 1640 & 1.89 \\
\cline { 2 - 5 } & Real & 870 & 1411 & 1.62 \\
\hline
\end{tabular}

these articles are suspicious. To obtain a small set of labeled samples, we first collect the news with reports and then send them to the experts of WeChat team for verification. Thus, the manually labeled fake and real news both have report messages. We split the fake news and real news into training and testing sets according to the post timestamp. The news in the training data were posted from March 2018 to September 2018, and testing dataset is from September 2018 to October 2018. There is no overlapped timestamp of news between these two sets. This design is to evaluate the performance of fake news detection on the fresh news. We also have an unlabeled set containing a large amount of collected news without annotation. The time window of the unlabeled set is from September to October 2018. The detailed statistics are shown in the Table 1. Note that the headlines can be seen as the summary of the news content. In the manual annotation process, experts only look at headlines to conduct labeling. Thus, in this paper, we use headlines as the input data.

\section{Baselines}

To validate the effectiveness of the proposed model, we choose both traditional machine learning algorithms and deep learning models as baseline methods. Previous work on verbal deception detection showed that LIWC (Pennebaker et al. 2015) is a valuable tool for the deception detection in various contexts (Ott et al. 2011; Pérez-Rosas et al. 2017). Based on LIWC features, we detect fake news with different traditional machine learning algorithms including Logistic Regression (LIWC-LR), SVM (LIWC-SVM) and Random Forest (LIWC-RF). Besides traditional machine learning algorithms, we also compare the proposed algorithm with the stat-of-the-art deep learning fake news detection models LSTM (Ruchansky, Seo, and Liu 2017), CNN (Wang et al. 2018) and EANN (Wang et al. 2018). To show effects of automatic annotation, we proposed two semi-supervised models based on CNN and LSTM introduced above, which are denoted as $\mathrm{LSTM}_{\text {semi }}$ and $\mathrm{CNN}_{\text {semi }}$ respectively, as baselines. Furthermore, the complete WeFEND model consists of three components: annotator, fake news detector and data selector. To show the role of data selector, we design one variant of the proposed model named WeFEND- , which does not include data selector.

Implementation Details. The 200 dimensional pre-trained word-embedding weights (Song et al. 2018) are used to initialize the parameters of the embedding layer. The architecture of the detector is the same as that of the baseline CNN. In the annotator, the weight $\mathbf{w}_{r} \in \mathbb{R}^{40 \times 20}$. In the reinforced selector, $\mathbf{w}_{s 1} \in \mathbb{R}^{88}$ and $\mathbf{w}_{s 2} \in \mathbb{R}^{8 \times 1}$. We set the bag size $B$ same as mini-batch size, $\tau=0.001$ and $K=200$. We implement all the deep learning baselines and the proposed framework with PyTorch 1.2. For training models, we use Adam (Kingma and $\mathrm{Ba}$ 2014) in the default setting. The learning rate $\alpha$ is 0.0001 . We use mini-batch size of 100 and training epochs of 100.

\section{Performance Comparison}

Table 2 shows the performance of different approaches on the WeChat dataset. We can observe that that the proposed framework achieves the best results in terms of Accuracy, AUC-ROC, precision, recall and $F_{1}$ measurement.

In the supervised setting, LIWC-LR achieves the worst performance. The reason is that LIWC-LR is a linear model and hard to discriminate the complicated distributions of fake and real news content. Compared with LIWC-LR, LIWC-SVM and LIWC-RN improve the performance in terms of most measurements. However, compared with traditional machine learning models, deep learning based models, including LSTM, CNN and EANN, significantly improve the performance. This confirms that deep learning models have superior ability to extract informative features for detection. In particular, compared with the best traditional machine learning baseline LIWC-RF, CNN achieves around 27\% and 35\% improvement on Accuracy and AUCROC respectively. EANN model has the ability to capture the dynamic nature of news by learning the event-invariant feature representations. It leads to the performance improvement and better generalization ability compared with the plain LSTM and CNN.

Along with the setting of supervised learning, in semisupervised setting, we incorporate external unlabeled news. We run LSTM and CNN models in the semi-supervised setting. Since the number of data largely increases, we can observe the performance improvement in both models. Take LSTM-based models as an example. The Accuracy and AUC-ROC of LSTM $_{\text {semi } i}$ increases 3\% and 5\% respectively, compared with supervised LSTM. This illustrates that using unlabeled data enlarges size of training set and achieves performance improvement.

The advantage of the proposed framework is that it can automatically annotate unlabeled news. From the results shown in Table 2, we can observe that the performance of WeFEND - is better than this of models in the supervised setting and semi-supervised setting.

Though incorporating automatic annotation as weak supervision helps fake news detection in some aspects, weak supervision is unavoidably noisy. In Table 2 , the recall values of WeFEND - improve as the coverage is increasing, but their precision values for fake news detection decrease. This shows that incorporating weak supervision may add more false positive examples. For real news, since the majority of unlabeled data with reports is still real news, the precision still improves. To reduce the influence of noisy labels, the proposed framework WeFEND has the data selector component based on reinforcement learning techniques. After incorporating data selector, the precision values of fake news and real news are improved compared with their reduced version in the same hybrid setting. Furthermore, we can observe from Table 2 that the proposed WeFEND achieve the best performance compared with all the baselines. 
Table 2: The performance comparison of different methods on WeChat dataset.

\begin{tabular}{c|c|c|c|c|c|c|c|c|c}
\hline \multirow{2}{*}{ Category } & \multirow{2}{*}{ Method } & \multirow{2}{*}{ Accuracy } & \multirow{2}{*}{ AUC-ROC } & \multicolumn{3}{|c|}{ Fake News } & \multicolumn{3}{|c}{ Real News } \\
\cline { 5 - 9 } & & & & Precision & Recall & $\mathrm{F}_{1}$ & Precision & Recall & $\mathrm{F}_{1}$ \\
\hline \multirow{5}{*}{ Supervised } & LIWC-LR & 0.528 & 0.558 & 0.604 & 0.160 & 0.253 & 0.517 & 0.896 & 0.655 \\
& LIWC-SVM & 0.568 & 0.598 & 0.574 & 0.521 & 0.546 & 0.563 & 0.614 & 0.587 \\
& LIWC-RF & 0.590 & 0.616 & 0.613 & 0.483 & 0.541 & 0.574 & 0.696 & 0.629 \\
& LSTM & 0.733 & 0.799 & 0.876 & 0.543 & 0.670 & 0.669 & $\mathbf{0 . 9 2 3}$ & 0.775 \\
& CNN & 0.747 & 0.834 & 0.869 & 0.580 & 0.696 & 0.685 & 0.913 & 0.783 \\
& EANN & 0.767 & 0.803 & 0.863 & 0.634 & 0.731 & 0.711 & 0.899 & 0.794 \\
\hline \multirow{2}{*}{ Semi-supervised } & LSTM $_{\text {semi }}$ & 0.753 & 0.841 & 0.854 & 0.611 & 0.713 & 0.697 & 0.895 & 0.784 \\
& CNN $_{\text {semi }}$ & 0.759 & 0.848 & 0.850 & 0.630 & 0.723 & 0.706 & 0.889 & 0.787 \\
\hline \multirow{2}{*}{ Automatically annotated } & WeFEND- $^{*}$ & 0.807 & 0.858 & 0.846 & $\mathbf{0 . 7 5 1}$ & 0.795 & 0.776 & 0.863 & 0.817 \\
& WeFEND $^{*}$ & $\mathbf{0 . 8 2 4}$ & $\mathbf{0 . 8 7 3}$ & $\mathbf{0 . 8 8 0}$ & $\mathbf{0 . 7 5 1}$ & $\mathbf{0 . 8 1 0}$ & $\mathbf{0 . 7 8 3}$ & 0.898 & $\mathbf{0 . 8 3 6}$ \\
\hline
\end{tabular}

\section{Insight Analysis}

Due to the dynamic nature of news, the annotation needs to be timely to cover news articles on newly emerged events. To address this issue, we propose to use reports from users to automatically annotate fresh news. To valid our intuition, we conduct experiments to demonstrate why reports are useful for this purpose.

The experiment is designed as follows. We first split the original training dataset consists of news content and reports into two sets: $80 \%$ data as the new training set (denoted as $D_{t}$ ) and the remaining $20 \%$ data as the testing set for the same time window setting (denoted as $D_{s}$ ). For the different time window setting, we randomly select a subset samples from original testing dataset, which is denoted as $D_{d}$. The number of samples in $D_{s}$ is similar as that in $D_{d}$. The fake news detector and annotator are first trained on the news content of $D_{t}$, and then we separately test the models on $D_{s}$ and $D_{d}$. We show the distributions of reports on two time sets. Since the real news is easy to collect, the goal of annotation procedure is to expand the size of fake news samples. Thus, to analyze the distribution of reports, we mainly focus on fake news samples. The distributions of reports on the same and different time set are shown in the Figure 2. For clear comparison between the distribution of reports and news content, the feature representations of news content for fake news are also shown in Figure 2a.

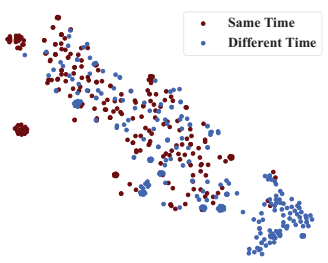

(a) News Content

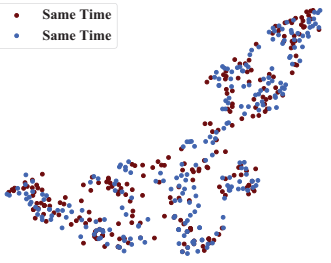

(b) Reports
Figure 2: The Visualization of latent representations for news content and reports of fake news.

From Figure 2a, we can observe that although the distributions of news content in the same time set and different time set have overlaps, the samples from two set are separately clustered at the top left and bottom right corner. This shows the distribution of news contents changes with time.
In contrast, the feature representations of report messages from two sets are all twisted and cannot be distinguished as shown in Figure 2b. This proves that the distributions of reports is time invariant and further explains why the model trained on report messages achieves a consistent performance. Thus, the annotation based on reports can guarantee consistent quality even for fresh news articles.

To demonstrate the importance of reinforced selector, we run WeFEND - ("w/o RL") and WeFEND ("w RL") 5 times, and the performance comparison during the first 30 epochs is shown in Figure 3. Note that the only difference between two models is whether it has the component of reinforced selector or not. The solid line represents the average accuracy of 5 times, and the line with light color represents the accuracy value of a single time. As the probability output from fake news detection model can provide more information for the reinforced selector, we can observe that the average accuracy of the model with reinforced selector is stably higher than that w/o reinforced selector after 12 epochs from Figure 3 . The ablation study shows that the designed reinforced selector is effective in improving the performance of fake news detection.

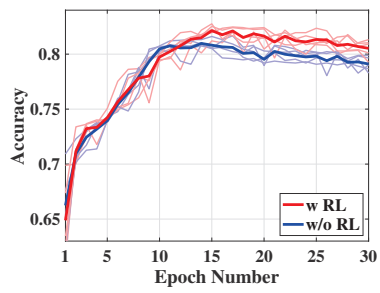

Figure 3: The changes of Accuracy in terms of the number of Epochs.

\section{Conclusions}

In this paper, we proposed to investigate the important problem of fake news detection. The dynamic nature of news make it infeasible to obtain continuously labeled highquality samples for training effective models, especially for training powerful deep learning based models. Therefore, we proposed a novel framework that can leverage user reports as weak supervision for fake news detection. The proposed framework works by integrating three components in- 
cluding the annotator, the reinforced selector and the fake news detector. The annotator automatically annotates unlabeled news articles as real or fake based on user reports. The reinforced selector based on reinforcement learning techniques chooses high-quality samples from those labeled by the annotator. The fake news detector then predicts the label of all the news articles by a model trained on the enhanced training set generated by both annotator and reinforced selector. By enhancing the quality and size of the training set, the proposed framework thus has shown significantly improved performance in fake news detection. This was demonstrated in a series of experiments conducted on a WeChat dataset consisting of news articles and user feedback.

\section{Acknowledgment}

The authors would like to thank the anonymous referees for their valuable comments and helpful suggestions. This work is supported in part by the US National Science Foundation under grant CNS-1742845. Any opinions, findings, and conclusions or recommendations expressed in this material are those of the author(s) and do not necessarily reflect the views of the National Science Foundation.

\section{References}

Castillo, C.; Mendoza, M.; and Poblete, B. 2011. Information credibility on twitter. In Proceedings of $W W W, 675-$ 684.

Chia, P. H., and Knapskog, S. J. 2011. Re-evaluating the wisdom of crowds in assessing web security. In Proceedings of FC, 299-314.

Conroy, N. J.; Rubin, V. L.; and Chen, Y. 2015. Automatic deception detection: Methods for finding fake news. Proceedings of the Association for Information Science and Technology 52(1):1-4.

Feng, J.; Huang, M.; Zhao, L.; Yang, Y.; and Zhu, X. 2018. Reinforcement learning for relation classification from noisy data. In Proceedings of AAAI.

Gupta, A.; Kumaraguru, P.; Castillo, C.; and Meier, P. 2014. Tweetcred: Real-time credibility assessment of content on twitter. In International Conference on Social Informatics, 228-243.

Jin, Z.; Cao, J.; Jiang, Y.-G.; and Zhang, Y. 2014. News credibility evaluation on microblog with a hierarchical propagation model. In Proceedings of ICDM, 230-239.

Kim, J.; Tabibian, B.; Oh, A.; Schölkopf, B.; and GomezRodriguez, M. 2018. Leveraging the crowd to detect and reduce the spread of fake news and misinformation. In Proceedings of WSDM, 324-332.

Kim, Y. 2014. Convolutional neural networks for sentence classification. arXiv preprint arXiv:1408.5882.

Kingma, D. P., and Ba, J. 2014. Adam: A method for stochastic optimization. arXiv preprint arXiv:1412.6980.

Kwon, S.; Cha, M.; Jung, K.; Chen, W.; and Wang, Y. 2013. Prominent features of rumor propagation in online social media. In Proceedings of ICDM, 1103-1108.
Ma, J.; Gao, W.; Mitra, P.; Kwon, S.; Jansen, B. J.; Wong, K.-F.; and Cha, M. 2016. Detecting rumors from microblogs with recurrent neural networks. In Proceedings of IJCAI, 3818-3824.

Moore, T., and Clayton, R. 2008. Evaluating the wisdom of crowds in assessing phishing websites. In Proceedings of $F C, 16-30$.

Ott, M.; Choi, Y.; Cardie, C.; and Hancock, J. T. 2011. Finding deceptive opinion spam by any stretch of the imagination. In Proceedings of ACL, 309-319.

Pennebaker, J. W.; Boyd, R. L.; Jordan, K.; and Blackburn, K. 2015. The development and psychometric properties of liwc2015. Technical report.

Pérez-Rosas, V.; Kleinberg, B.; Lefevre, A.; and Mihalcea, R. 2017. Automatic detection of fake news. arXiv preprint arXiv:1708.07104.

Popat, K.; Mukherjee, S.; Yates, A.; and Weikum, G. 2018. Declare: Debunking fake news and false claims using evidence-aware deep learning. arXiv preprint arXiv:1809.06416.

Ruchansky, N.; Seo, S.; and Liu, Y. 2017. Csi: A hybrid deep model for fake news detection. In Proceedings of CIKM, 797-806.

Shen, H.; Ma, F.; Zhang, X.; Zong, L.; Liu, X.; and Liang, W. 2017. Discovering social spammers from multiple views. Neurocomputing 225:49-57.

Shu, K.; Sliva, A.; Wang, S.; Tang, J.; and Liu, H. 2017. Fake news detection on social media: A data mining perspective. ACM SIGKDD Explorations Newsletter 19(1):22-36.

Song, Y.; Shi, S.; Li, J.; and Zhang, H. 2018. Directional skip-gram: Explicitly distinguishing left and right context for word embeddings. In Proceedings of NAACL, 175-180.

Sutton, R. S., and Barto, A. G. 1998. Introduction to reinforcement learning, volume 135. MIT press Cambridge.

Sutton, R. S.; McAllester, D. A.; Singh, S. P.; and Mansour, Y. 2000. Policy gradient methods for reinforcement learning with function approximation. In Proceedings of NIPS, 1057-1063.

Tacchini, E.; Ballarin, G.; Della Vedova, M. L.; Moret, S.; and de Alfaro, L. 2017. Some like it hoax: Automated fake news detection in social networks. arXiv preprint arXiv:1704.07506.

Tschiatschek, S.; Singla, A.; Gomez Rodriguez, M.; Merchant, A.; and Krause, A. 2018. Fake news detection in social networks via crowd signals. In Companion Proceedings of the The Web Conference, 517-524.

Wang, Y.; Ma, F.; Jin, Z.; Yuan, Y.; Xun, G.; Jha, K.; Su, L.; and Gao, J. 2018. Eann: Event adversarial neural networks for multi-modal fake news detection. In Proceedings of KDD, 849-857.

Wu, J.; Li, L.; and Wang, W. Y. 2018. Reinforced cotraining. arXiv preprint arXiv:1804.06035.

Wu, K.; Yang, S.; and Zhu, K. Q. 2015. False rumors detection on sina weibo by propagation structures. In Proceedings of ICDE, 651-662. 